\title{
Extending the lifetime of 3D black hole computations with a new hyperbolic system of evolution equations
}

\author{
Lawrence E. Kidder, Mark A. Scheel, and Saul A. Teukolsky \\ Center for Radiophysics and Space Research, Cornell University, Ithaca, New York 14853
}

(Received 8 May 2001; published 27 August 2001)

\begin{abstract}
We present a new many-parameter family of hyperbolic representations of Einstein's equations, which we obtain by a straightforward generalization of previously known systems. We solve the resulting evolution equations numerically for a Schwarzschild black hole in three spatial dimensions, and find that the stability of the simulation is strongly dependent on the form of the equations (i.e. the choice of parameters of the hyperbolic system), independent of the numerics. For an appropriate range of parameters we can evolve a single three-dimensional black hole to $t \simeq 600 M-1300 M$, and we are apparently limited by constraint-violating solutions of the evolution equations. We expect that our method should result in comparable times for evolutions of a binary black hole system.
\end{abstract}

DOI: 10.1103/PhysRevD.64.064017

PACS number(s): 04.25.Dm, 02.70.Hm

\section{INTRODUCTION}

A key unsolved problem in general relativity is to provide a detailed description of the final moments of a binary black hole system as the two black holes plunge together and merge. While this problem is interesting in its own right, the current deployment of the Laser Interferometric Gravitational Wave Observatory and other gravitational wave interferometers provide additional incentive for finding a timely solution: coalescing compact binaries are expected to be the primary sources of gravitational radiation observable by these instruments. A comparison of observed gravitational wave forms to detailed theoretical predictions of binary black hole evolution may allow one to test general relativity and other theories of gravitation, to identify black holes in distant galaxies and to measure their masses and spins.

Although both the initial inspiral of a binary black hole system and the final ringdown of the resulting Kerr black hole are well described by perturbation theory, understanding the plunge from the innermost stable quasicircular orbit through the coalescence will require numerical solutions of the full Einstein equations in three spatial dimensions. Such numerical computations are in progress $[1,2]$; however, they are currently plagued with instabilities that severely limit the duration of the simulations. Indeed, until recently [3] threedimensional (3D) Cauchy evolution codes without built-in symmetries have had great difficulty evolving even a single Schwarzschild black hole for the amount of time that would be required for a binary orbit.

Many of the stability difficulties affecting black hole computations are undoubtedly due to the technical details of the numerical solution scheme; there are many such difficulties to overcome in any large scale numerical solution of partial differential equations. However, there is also evidence that some of the stability problems are due to properties of the equations themselves, independent of any numerical approximation. In particular, by rewriting the equations in a different manner but leaving the numerical method unmodified, one can significantly affect the stability of the computation [2-7].
Einstein's equations, when written as a Cauchy problem, can be decomposed into two subsystems of equations: constraint equations that must be obeyed on each spacelike hypersurface, or time slice, and evolution equations that describe how quantities propagate from one hypersurface to the next. An analogous decomposition occurs in electromagnetism, which is naturally split into time-independent (divergence) equations that constrain the fields at a particular time, and time-dependent (curl) equations that determine their evolution. For both electromagnetism and gravitation, the system of equations is overdetermined in the following sense: if the constraint equations are satisfied at some initial time, then the evolution equations guarantee that they will be satisfied at subsequent times. For numerical black hole computations, one typically solves the constraint equations only on the initial time slice, and then uses the evolution equations to advance the solution in time.

However, the decomposition of Einstein's equations into evolution equations and constraints is not unique. For example, one can add any combination of constraints to any of the evolution equations to produce a different decomposition. Indeed, there have been a large number of new formulations of $3+1$ general relativity proposed in recent years [5,8-27], many of which have attractive properties such as symmetric hyperbolicity.

All such formulations must have the same physical solutions since they describe the same underlying theory. However, the set of evolution equations also admits unphysical solutions such as constraint-violating modes, and these unphysical solutions will be different for each formulation. Usually one is not interested in unphysical solutions, but if such a solution grows rapidly with time, any small perturbation (say, caused by numerical errors) that excites this solution will grow and eventually overwhelm the physical solution. This is one reason why some formulations of Einstein's equations may be better suited for numerical evolution than others.

In order to explore the extent to which different formulations of Einstein's equations affect the stability of numerical evolutions, we construct three new formulations of Einstein's equations, following a method similar to that of Ref. [13]. 
(1) A first-order system obtained directly from the Arnowitt-Deser-Misner (ADM) [28] system. This system has five undetermined constant parameters that specify constraint terms to be added to the evolution equations. These parameters determine the hyperbolicity of the evolution equations and the values of the characteristic speeds. We find that constraining the system to have physical characteristic speeds (i.e., the characteristic fields propagate either along the light cone or normal to the time slice) still leaves two of the five parameters free, and guarantees that the evolution equations are strongly hyperbolic. In this case, the constraint quantities also evolve in a strongly hyperbolic manner with physical characteristic speeds.

(2) A 12-parameter system obtained by applying a parameterized change of variables to system (1). The additional seven parameters are completely free, and do not affect the hyperbolicity of either the evolution equations or the evolution of the constraint quantities. This system can be reduced to either the Frittelli-Reula formulation [13] or the EinsteinChristoffel formulation [22] with an appropriate choice of parameters. The seven additional parameters can be used either to simplify the equations or to improve the numerical behavior of the system.

(3) A two-parameter system that is obtained from system (2) by demanding that the principal part of the equations is equivalent to a scalar wave equation for each of the six components of $g_{i j}$. This system is particularly simple, is symmetrizable hyperbolic with physical characteristic speeds, and includes the Einstein-Christoffel formulation [22] as a special case.

To determine whether modifying the formulation significantly effects the numerical solution of the evolution equations, we perform numerical evolutions of single black holes using a new 3D code we have developed. We evolve system (3) for simplicity. We find that by varying the two parameters in system (3) while keeping the numerical evolution method fixed, we can vary the run time of the simulation by more than an order of magnitude. For a single black hole, our optimum choice of parameters yields evolutions that run to $t=600 M-1300 M$. This is long enough that, if this result carries over to two-black-hole simulations, one could simulate the last few orbits of a binary system and the final merger.

In Sec. II we derive systems 1-3 and conditions for hyperbolicity. We also derive evolution equations for the constraint quantities and discuss their hyperbolicity. In Sec. III we present numerical evolutions of system (3) for different choices of parameters, and show that particular choices yield significant improvements. In Sec. IV we discuss our results and our plans to simulate a binary system.

\section{PARAMETRIZED HYPERBOLIC SYSTEM}

\section{A. 3+1 ADM}

We begin with the standard $3+1$ formulation of Ref. [28], which was discussed in detail in Ref. [29]. Fourdimensional spacetime is foliated by the level surfaces $\Sigma_{t}$ of a function $t\left(x^{\mu}\right)$. Let $n^{\mu}$ be the unit normal vector to the hypersurfaces $\Sigma_{t}$. Then the spacetime metric ${ }^{(4)} g_{\mu \nu}$ induces the spatial 3-metric $g_{\mu \nu}$ on each $\Sigma_{t}$ given by

$$
g_{\mu \nu}={ }^{(4)} g_{\mu \nu}+n_{\mu} n_{\nu} .
$$

The timelike vector $t^{\mu}$ is defined such that $t^{\mu} t_{; \mu}=1$, where $t_{; \mu}$ is the covariant derivative of $t$ with respect to the spacetime metric. The lapse function $N$ and shift vector $\beta^{\mu}$ are defined by

$$
\begin{gathered}
N \equiv-t^{\mu} n_{\mu}, \\
\beta_{\mu} \equiv g_{\mu \nu} t^{\nu} .
\end{gathered}
$$

If we adopt a coordinate system $\left\{t, x^{i}\right\}$ adapted to the spatial hypersurfaces, the line element is given in the usual $3+1$ form

$$
d s^{2}=-N^{2} d t^{2}+g_{i j}\left(d x^{i}+\beta^{i} d t\right)\left(d x^{j}+\beta^{j} d t\right) .
$$

The extrinsic curvature $K_{i j}$ of the spatial surfaces is given by

$$
K_{i j}=-\frac{1}{2} £_{n} g_{i j},
$$

where $£$ denotes a Lie derivative.

Einstein's equations are given in covariant form by

$$
{ }^{(4)} R_{\mu \nu}-\frac{1}{2}{ }^{(4)} g_{\mu \nu}{ }^{(4)} R=8 \pi T_{\mu \nu},
$$

where ${ }^{(4)} R_{\mu \nu}$ and ${ }^{(4)} R$ are the Ricci tensor and Ricci scalar associated with the spacetime metric, and $T_{\mu \nu}$ is the stressenergy tensor. In the $3+1$ decomposition Einstein's equations are decomposed into the Hamiltonian constraint

$$
\mathcal{C} \equiv \frac{1}{2}\left(R-K_{a b} K^{a b}+K^{2}\right)-8 \pi \rho=0,
$$

the momentum constraints

$$
\mathcal{C}_{i} \equiv \nabla_{a} K_{i}^{a}-\nabla_{i} K-8 \pi J_{i}=0,
$$

and the evolution equations

$$
\begin{aligned}
\hat{\partial}_{0} K_{i j}= & -\nabla_{i} \nabla_{j} N+N R_{i j}-2 N K_{i a} K_{j}^{a}+N K K_{i j}-8 \pi N S_{i j} \\
& -4 \pi N g_{i j}(\rho-S),
\end{aligned}
$$

where $K=g^{a b} K_{a b}$, and $\nabla_{i}, R_{i j}$, and $R$ are the covariant derivative, Ricci tensor, and Ricci scalar associated with the spatial 3-metric. The symbol $\hat{\partial}_{0}$ is the time derivative operator normal to the spatial foliation, defined by

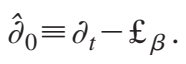

The matter terms are defined as 


$$
\begin{gathered}
\rho \equiv n^{\mu} n^{\nu} T_{\mu \nu}, \\
J_{i} \equiv-n^{\mu} g_{i}{ }^{\nu} T_{\mu \nu}, \\
S_{i j} \equiv g_{i}{ }^{\nu} g_{j}{ }^{\mu} T_{\mu \nu},
\end{gathered}
$$

and $S=g^{a b} S_{a b}$. Definition (2.5) of the extrinsic curvature yields the following evolution equation for the spatial metric:

$$
\hat{\partial}_{0} g_{i j}=-2 N K_{i j}
$$

Note that the spatial metric and its inverse are used to lower and raise the indices of all spatial tensors.

\section{B. First-order form}

In order to cast the evolution equations in first-order form, we must eliminate the second derivatives of the spatial metric. We define a new variable (symmetric on its last two indices)

$$
d_{k i j} \equiv \partial_{k} g_{i j}
$$

and its traces $d_{k} \equiv g^{a b} d_{k a b}$ and $b_{k} \equiv g^{a b} d_{a b k}$. An evolution equation for $d_{k i j}$ is obtained by taking a spatial derivative of Eq. (2.12) and using the fact that $\partial_{k}$ and $\hat{\partial}_{0}$ commute. This yields

$$
\hat{\partial}_{0} d_{k i j}=-2 N \partial_{k} K_{i j}-2 K_{i j} \partial_{k} N
$$

where the Lie derivative of $d_{k i j}$ is

$$
£_{\beta} d_{k i j}=\beta^{a} \partial_{a} d_{k i j}+d_{a i j} \partial_{k} \beta^{a}+2 d_{k a(i} \partial_{j)} \beta^{a}+2 g_{a(i} \partial_{j)} \partial_{k} \beta^{a} .
$$

Since we have introduced a new variable that we will evolve independently of the metric, we have an additional constraint

$$
\mathcal{C}_{k i j} \equiv d_{k i j}-\partial_{k} g_{i j}=0,
$$

which must be satisfied in order for a solution of the firstorder evolution equations to be a solution of Einstein's equations. Note that the spatial derivatives of $d_{k i j}$ must satisfy the constraint

$$
\mathcal{C}_{k l i j} \equiv \partial_{[k} d_{l] i j}=0
$$

because second derivatives of the metric commute. Therefore we make the following substitution when we encounter second derivatives of the metric:

$$
\partial_{k} \partial_{l} g_{i j}=\partial_{(k} d_{l) i j}
$$

In terms of these new variables, the affine connection, Ricci tensor, and Ricci scalar are given by

$$
\begin{aligned}
\Gamma_{k i j}= & d_{(i j) k}-\frac{1}{2} d_{k i j}, \\
R_{i j}= & \frac{1}{2} g^{a b}\left(\partial_{(i} d_{a b j)}+\partial_{a} d_{(i j) b}-\partial_{a} d_{b i j}-\partial_{(i} d_{j) a b}\right) \\
& +\frac{1}{2} b^{a} d_{a i j}-\frac{1}{4} d^{a} d_{a i j}-b^{a} d_{(i j) a}-\frac{1}{2} d_{a j}{ }^{b} d_{b i}{ }^{a} \\
& +\frac{1}{2} d^{a} d_{(i j) a}+\frac{1}{4} d_{i}{ }^{a b} d_{j a b}+\frac{1}{2} d^{a b}{ }_{i} d_{a b j}, \\
R= & g^{a b} g^{c d}\left(\partial_{d} d_{a b c}-\partial_{a} d_{b c d}\right)+b^{a} d_{a}-b_{a} b^{a}-\frac{1}{4} d_{a} d^{a} \\
& -\frac{1}{2} d_{a b c} d^{c a b}+\frac{3}{4} d_{a b c} d^{a b c} .
\end{aligned}
$$

The constraint equations are given by

$$
\begin{aligned}
\mathcal{C}= & \frac{1}{2} g^{a b} g^{c d}\left(\partial_{d} d_{a b c}-\partial_{a} d_{b c d}\right)+\frac{1}{2} b^{a} d_{a}-\frac{1}{2} b_{a} b^{a}-\frac{1}{8} d_{a} d^{a} \\
& -\frac{1}{4} d_{a b c} d^{c a b}+\frac{3}{8} d_{a b c} d^{a b c}-\frac{1}{2} K_{a b} K^{a b}+\frac{1}{2} K^{2}-8 \pi \rho \\
\mathcal{C}_{i}= & g^{a b}\left(\partial_{a} K_{i b}-\partial_{i} K_{a b}\right)+\frac{1}{2} K^{a b} d_{i a b}+\frac{1}{2} K_{i a} d^{a}-K_{i a} b^{a} \\
& -8 \pi J_{i} .
\end{aligned}
$$

Finally, the evolution equation for the extrinsic curvature becomes

$$
\begin{aligned}
\hat{\partial}_{0} K_{i j}= & N\left[\frac{1}{2} g^{a b}\left(\partial_{(i} d_{a b j)}+\partial_{a} d_{(i j) b}-\partial_{a} d_{b i j}-\partial_{(i} d_{j) a b}\right)\right. \\
& +\frac{1}{2} b^{a} d_{a i j}-\frac{1}{4} d^{a} d_{a i j}-b^{a} d_{(i j) a}-\frac{1}{2} d_{a j}{ }^{b} d_{b i}{ }^{a} \\
& +\frac{1}{2} d^{a} d_{(i j) a}+\frac{1}{4} d_{i}^{a b} d_{j a b}+\frac{1}{2} d^{a b}{ }_{i} d_{a b j} \\
& \left.-2 K_{i a} K_{j}{ }^{a}+K K_{i j}\right]-\partial_{i} \partial_{j} N-\frac{1}{2} d^{a}{ }_{i j} \partial_{a} N \\
& +d_{(i j)}{ }^{a} \partial_{a} N-8 \pi N S_{i j}-4 \pi N g_{i j}(\rho-S) .
\end{aligned}
$$

The hyperbolicity of the system of evolution equations can be determined by examining its principal part. Consider a system of the form

$$
\hat{\partial}_{0} u+A^{i} \partial_{i} u=F,
$$

where $u$ is a column vector of the fundamental variables, and $A^{i}$ and $F$ are matrices that can depend on $u$ but not on derivatives of $u$. For a particular unit 1-form $\xi_{i}$, one defines a characteristic matrix $C$ in the direction normal to $\xi_{i}$ :

$$
C \equiv A^{i} \xi_{i} .
$$


The characteristic speeds in the direction $\xi_{i}$ are the eigenvalues of $C$. If all characteristic speeds are real, then the system is said to be weakly hyperbolic. If, in addition, $C$ has a complete set of eigenvectors, and the matrix of these eigenvectors and its inverse are uniformly bounded functions of $\xi_{i}$, the spacetime coordinates, and the solution, then the system is said to be strongly hyperbolic. If the matrices $A^{i}$ are symmetric, the system is said to be symmetric hyperbolic. If the matrices $A^{i}$ can be brought into a symmetric form by multiplying by a positive-definite matrix called a symmetrizer, the system is said to be symmetrizable hyperbolic. Symmetric, symmetrizable, and strongly hyperbolic systems admit a well-posed Cauchy problem; weakly hyperbolic systems do not [30].

For the systems described in this paper, we explicitly construct a complete set of eigenvectors that depend upon $\xi_{i}$, the metric, and its inverse. Provided that the matrix norms of the metric and its inverse remain bounded, then the norms of the matrix of eigenvectors and its inverse are bounded, so the system is strongly hyperbolic [31].

Using the method outlined in Appendix A, and assuming that the lapse $N$ and shift vector $\beta^{i}$ are arbitrary gauge functions independent of the dynamical variables, we find that the ADM equations written in first-order form are only weakly hyperbolic, as the characteristic matrix of the system has eigenvalues $\{0, \pm 1\}$, but does not have a complete set of eigenvectors. Fortunately, the hyperbolicity of the equations can be changed by "densitizing" the lapse and adding constraints to the evolution equations.

\section{Densitization of the lapse}

We densitize the lapse by defining

$$
Q \equiv \log \left(N g^{-\sigma}\right),
$$

where $g$ is the determinant of the 3-metric, and $\sigma$ is the densitization parameter, which is an arbitrary constant. The lapse density $Q$ and the shift vector $\beta^{i}$ will be considered as arbitrary gauge functions independent of the dynamical fields. With this definition we have

$$
\begin{aligned}
\partial_{i} N= & N\left(\partial_{i} Q+\sigma d_{i}\right), \\
\partial_{i} \partial_{j} N= & N\left[\partial_{i} \partial_{j} Q+\left(\partial_{i} Q\right)\left(\partial_{j} Q\right)+2 \sigma d_{(i} \partial_{j)} Q\right. \\
& \left.+\sigma g^{a b} \partial_{(i} d_{j) a b}-\sigma d_{i a b} d_{j}^{a b}+\sigma^{2} d_{i} d_{j}\right] .
\end{aligned}
$$

Substituting the above expressions into the evolution equations, and examining the hyperbolicity of the modified evolution equations, we find that densitizing the lapse is not sufficient to make the evolution system strongly hyperbolic. In order for the system to remain even weakly hyperbolic the densitization parameter must satisfy $\sigma \geqslant 0$, as the eigenvalues of the characteristic matrix are now $\{0, \pm 1, \pm \sqrt{2 \sigma}\}$. In Sec. II D, we will find that densitizing the lapse is a necessary condition for strong hyperbolicity, and that if we demand physical characteristic speeds we must choose $\sigma=\frac{1}{2}$.

\section{Addition of constraints: System (1)}

By adding terms proportional to the constraints, we can modify the evolution equations for $K_{i j}$ and $d_{k i j}$ without affecting the physical solution. We modify the evolution equations (2.14) and (2.24) by

$$
\begin{gathered}
\hat{\partial}_{0} K_{i j}=(\cdots)+\gamma N g_{i j} \mathcal{C}+\zeta N g^{a b} \mathcal{C}_{a(i j) b}, \\
\hat{\partial}_{0} d_{k i j}=(\cdots)+\eta N g_{k(i} \mathcal{C}_{j)}+\chi N g_{i j} \mathcal{C}_{k},
\end{gathered}
$$

where $(\cdots)$ represents the right-hand side of either equation (2.14) or (2.24), and the constraint parameters $\{\gamma, \zeta, \eta, \chi\}$ are arbitrary constants. The evolution equations are now given by

$$
\begin{aligned}
\hat{\partial}_{0} g_{i j} \simeq & 0 \\
\hat{\partial}_{0} K_{i j} \simeq & -\frac{1}{2} N g^{a b}\left[\partial_{a} d_{b i j}-(1+\zeta) \partial_{a} d_{(i j) b}-(1-\zeta) \partial_{(i} d_{a b j)}\right. \\
& +(1+2 \sigma) \partial_{(i} d_{j) a b}-\gamma g_{i j} g^{c d} \partial_{a} d_{c d b} \\
& \left.+\gamma g_{i j} g^{c d} \partial_{a} d_{b c d}\right], \\
\hat{\partial}_{0} d_{k i j} \simeq & -2 N \partial_{k} K_{i j}+N g^{a b}\left(\eta g_{k(i} \partial_{a} K_{b j)}+\chi g_{i j} \partial_{a} K_{b k}\right. \\
& \left.-\eta g_{k(i} \partial_{j)} K_{a b}-\chi g_{i j} \partial_{k} K_{a b}\right),
\end{aligned}
$$

where $\simeq$ denotes equal to the principal part. For brevity, we show only the principal parts of the evolution equations, as these are what determine the hyperbolicity of the system. The full evolution equations are lengthy and available from the authors upon request.

We find that the eigenvalues of the characteristic matrix of the system are $\left\{0, \pm 1, \pm c_{1}, \pm c_{2}, \pm c_{3}\right\}$, where

$$
\begin{aligned}
& c_{1}=\sqrt{2 \sigma}, \\
& c_{2}=\frac{1}{2 \sqrt{2}} \sqrt{\eta-4 \eta \sigma-2 \chi-12 \sigma \chi-3 \eta \zeta}, \\
& c_{3}=\frac{1}{\sqrt{2}} \sqrt{2+4 \gamma-\eta-2 \gamma \eta+2 \chi+4 \gamma \chi-\eta \zeta} .
\end{aligned}
$$

Thus, in order for the system to be weakly hyperbolic, the parameters must satisfy

$$
\begin{aligned}
& \sigma \geqslant 0, \\
& \eta-4 \eta \sigma-2 \chi-12 \sigma \chi-3 \eta \zeta \geqslant 0, \\
& 2+4 \gamma-\eta-2 \gamma \eta+2 \chi+4 \gamma \chi-\eta \zeta \geqslant 0 .
\end{aligned}
$$

If the above conditions are met, we find a complete set of eigenvectors, so that the system is strongly hyperbolic, unless one of the following conditions occur:

$$
c_{i}=0 \text {, }
$$




$$
\begin{aligned}
& c_{1}=c_{3} \neq 1, \\
& c_{1}=c_{3}=1 \neq c_{2} .
\end{aligned}
$$

If any of the above conditions are met, the system is only weakly hyperbolic. Note that if $\sigma=0$, then $c_{1}=0$, so that densitizing the lapse is a necessary condition for strong hyperbolicty. Also note that if $\eta=\chi=0$, then $c_{2}=0$, so that constraints must be added to the evolution equation for $d_{k i j}$ in order to have a strongly hyperbolic system.

For physical characteristic speeds, each of the $c_{i}$ 's is either zero or unity. To make them all unity (the only choice that yields strongly hyperbolic evolution equations) requires either

$$
\begin{aligned}
& \sigma=1 / 2, \\
& \zeta=-\frac{8+5 \eta+10 \gamma \eta}{\eta(7+6 \gamma)}, \\
& \chi=-\frac{4+6 \gamma-\eta-3 \gamma \eta}{(7+6 \gamma)}
\end{aligned}
$$

or

$$
\{\sigma, \gamma, \zeta, \eta, \chi\}=\left\{\frac{1}{2},-\frac{7}{6},-\frac{1}{9}(23+20 \chi), \frac{6}{5}, \chi\right\}
$$

In the first case, there are two free parameters, and in the second case there is one. In both cases, the evolution equations are strongly hyperbolic as long as the free parameters are chosen such that all five parameters are finite.

\section{E. Evolution of the constraints}

Taking $\hat{\partial}_{0}$ of the constraints, and replacing all derivatives of the fundamental variables with the constraints and their spatial derivatives, we obtain the following equations for the evolution of the constraints:

$$
\begin{aligned}
\hat{\partial}_{0} \mathcal{C} \simeq & -\frac{1}{2}(2-\eta+2 \chi) N g^{p q} \partial_{p} \mathcal{C}_{q} \\
\hat{\partial}_{0} \mathcal{C}_{i} \simeq & -(1+2 \gamma) N \partial_{i} \mathcal{C}+\frac{1}{2} N g^{p q} g^{r s}\left[(1-\zeta) \partial_{q} \mathcal{C}_{p r s i}\right. \\
& \left.+(1+\zeta) \partial_{p} \mathcal{C}_{s i q r}-(1+2 \sigma) \partial_{p} \mathcal{C}_{q i r s}\right] \\
\hat{\partial}_{0} \mathcal{C}_{k i j} \simeq & 0 \\
\hat{\partial}_{0} \mathcal{C}_{k l i j}= & \frac{1}{2} \eta N\left(g_{j[l} \partial_{k]} \mathcal{C}_{i}+g_{i[l} \partial_{k]} \mathcal{C}_{j}\right)+\chi N g_{i j} \partial_{[k} \mathcal{C}_{l]}
\end{aligned}
$$

where again for brevity we have only shown the principal parts of the equations.

The eigenvalues for the constraint evolution system are $\left\{0, \pm c_{2}, \pm c_{3}\right\}$. Because this is a subset of the eigenvalues of the evolution equations, the constraints will propagate at the same speeds as some of the characteristic fields of the evolved quantities. Furthermore, we find that the constraint evolution system is strongly hyperbolic whenever the regular evolution system is strongly hyperbolic.

\section{F. Redefining the variables: System (2)}

The evolution equations can also be modified by redefining the variables that are evolved. We define the generalized extrinsic curvature $P_{i j}$ using the relation

$$
P_{i j} \equiv K_{i j}+\hat{z} g_{i j} K
$$

where $\hat{z}$ is an arbitrary parameter. The inverse transformation is given by

$$
K_{i j}=P_{i j}+\bar{z} g_{i j} P
$$

where $P \equiv g^{a b} P_{a b}$, and

$$
\bar{z}=-\frac{\hat{z}}{1+3 \hat{z}}
$$

which implies that $\hat{z} \neq-\frac{1}{3}$ for the inverse transformation to exist.

We define the generalized derivative of the metric, $M_{k i j}$, using the relation

$$
\begin{aligned}
M_{k i j}= & \frac{1}{2}\left\{\hat{k} d_{k i j}+\hat{e} d_{(i j) k}+g_{i j}\left[\hat{a} d_{k}+\hat{b} b_{k}\right]\right. \\
& \left.+g_{k(i}\left[\hat{c} d_{j)}+\hat{d} b_{j)}\right]\right\} .
\end{aligned}
$$

The inverse transformation is given by

$$
\begin{aligned}
d_{k i j}= & 2\left\{\bar{k} M_{k i j}+\bar{e} M_{(i j) k}+g_{i j}\left[\bar{a} M_{k}+\bar{b} W_{k}\right]\right. \\
& \left.+g_{k(i}\left[\bar{c} M_{j)}+\bar{d} W_{j)}\right]\right\},
\end{aligned}
$$

where the traces $M_{k} \equiv g^{a b} M_{k a b}$ and $W_{k} \equiv g^{a b} M_{a b k}$, and

$$
\begin{aligned}
\delta \bar{a}= & 6 \hat{b} \hat{c} \hat{e}-6 \hat{a} \hat{d} \hat{e}-\hat{a} \hat{e}^{2}+\hat{b} \hat{e}^{2}+\hat{c} \hat{e}^{2}-\hat{d} \hat{e}^{2}+8 \hat{b} \hat{c} \hat{k}-8 \hat{a} \hat{d} \hat{k} \\
& -4 \hat{a} \hat{e} \hat{k}+2 \hat{b} \hat{e} \hat{k}+2 \hat{c} \hat{e} \hat{k}-4 \hat{a} \hat{k}^{2}, \\
\delta \bar{b}= & -8 \hat{b} \hat{c} \hat{e}+8 \hat{a} \hat{d} \hat{e}+2 \hat{a} \hat{e}^{2}-2 \hat{c} \hat{e}^{2}-4 \hat{b} \hat{c} \hat{k}+4 \hat{a} \hat{d} \hat{k}+4 \hat{a} \hat{e} \hat{k} \\
& -2 \hat{b} \hat{e} \hat{k}+2 \hat{d} \hat{e} \hat{k}-4 \hat{b} \hat{k}^{2}, \\
\delta \bar{c}= & -8 \hat{b} \hat{c} \hat{e}+8 \hat{a} \hat{d} \hat{e}+2 \hat{a} \hat{e}^{2}-2 \hat{b} \hat{e}^{2}-4 \hat{b} \hat{c} \hat{k}+4 \hat{a} \hat{d} \hat{k}+4 \hat{a} \hat{e} \hat{k} \\
& -2 \hat{c} \hat{e} \hat{k}+2 \hat{d} \hat{e} \hat{k}-4 \hat{c} \hat{k}^{2}, \\
\delta \bar{d}= & 4 \hat{b} \hat{c} \hat{e}-4 \hat{a} \hat{d} \hat{e}-4 \hat{a} \hat{e}^{2}+12 \hat{b} \hat{c} \hat{k}-12 \hat{a} \hat{d} \hat{k}+4 \hat{b} \hat{e} \hat{k}+4 \hat{c} \hat{e} \hat{k} \\
& -4 \hat{d} \hat{k}^{2}, \\
\delta_{0} \bar{e}= & 2 \hat{e}, \\
\delta_{0} \bar{k}= & -\hat{e}-2 \hat{k},
\end{aligned}
$$




$$
\begin{aligned}
\delta_{0}= & \hat{e}^{2}-\hat{e} \hat{k}-2 \hat{k}^{2} \\
\delta= & \delta_{0}\left(10 \hat{b} \hat{c}-10 \hat{a} \hat{d}-\hat{a} \hat{e}+3 \hat{b} \hat{e}+3 \hat{c} \hat{e}+\hat{d} \hat{e}+\hat{e}^{2}-6 \hat{a} \hat{k}\right. \\
& \left.-2 \hat{b} \hat{k}-2 \hat{c} \hat{k}-4 \hat{d} \hat{k}-\hat{e} \hat{k}-2 \hat{k}^{2}\right)
\end{aligned}
$$

For the inverse transformation to exist, $\delta \neq 0$.

Thus we have seven additional redefinition parameters $\{\hat{a}, \hat{b}, \hat{c}, \hat{d}, \hat{e}, \hat{k}, \hat{z}\}$ (or equivalently $\{\bar{a}, \bar{b}, \bar{c}, \bar{d}, \bar{e}, \bar{k}, \bar{z}\}$ ) that can be used to modify the evolution equations. Note that Eqs. (2.46) and (2.49) remain true under interchange of $\{\hat{a}, \hat{b}, \hat{c}, \hat{d}, \hat{e}, \hat{k}, \hat{z}\}$ and $\{\bar{a}, \bar{b}, \bar{c}, \bar{d}, \bar{e}, \bar{k}, \bar{z}\}$.

When the principal terms in system (1) are transformed, terms containing derivatives of the metric appear because of the traces in Eqs. (2.45) and (2.48). These terms are eliminated using Eqs. (2.12) and (2.13).

The redefinition parameters do not change the eigenvalues of the evolution system, nor do they change whether or not the system is strongly hyperbolic (see Appendix B). In addition, they have no effect on the principal part of the constraint evolution equations. The redefinition parameters, however, do affect the eigenvectors of the evolution system and thus also affect the characteristic fields. In addition, the redefinition parameters change the nonlinear terms in the nonprincipal parts of the evolution equations and the constraint evolution system.

The principal parts of the evolution equations for $P_{i j}$ and $M_{k i j}$ are

$$
\begin{aligned}
\hat{\partial}_{0} g_{i j} \simeq & 0 \\
\hat{\partial}_{0} P_{i j} \simeq & -N g^{a b}\left(\mu_{1} \partial_{a} M_{b i j}+\mu_{2} \partial_{a} M_{(i j) b}+\mu_{3} \partial_{(i} M_{a b j)}\right. \\
& +\mu_{4} \partial_{(i} M_{j) a b}+\mu_{5} g_{i j} g^{c d} \partial_{a} M_{c d b} \\
& \left.+\mu_{6} g_{i j} g^{c d} \partial_{a} M_{b c d}\right) \\
\hat{\partial}_{0} M_{k i j} \simeq & -N\left(\nu_{1} \partial_{k} P_{i j}+\nu_{2} \partial_{i} P_{j) k}+\nu_{3} g^{a b} g_{k(i} \partial_{a} P_{b j)}\right. \\
& +\nu_{4} g_{i j} g^{a b} \partial_{a} P_{b k}+\nu_{5} g^{a b} g_{k(i} \partial_{j)} P_{a b} \\
& \left.+\nu_{6} g_{i j} g^{a b} \partial_{k} P_{a b}\right)
\end{aligned}
$$

where

$$
\begin{aligned}
\mu_{1}= & \bar{k}-\frac{1}{2}(1+\zeta) \bar{e} \\
\mu_{2}= & \frac{1}{2}(1-\zeta) \bar{e}-(1+\zeta) \bar{k}, \\
\mu_{3}= & (1+6 \sigma) \bar{b}-(1-\zeta) \bar{k}-\frac{1}{2}(1-4 \sigma-3 \zeta) \bar{d} \\
& +\frac{1}{2}(1+4 \sigma+\zeta) \bar{e},
\end{aligned}
$$

$$
\begin{aligned}
\mu_{4}= & (1+6 \sigma) \bar{a}+(1+2 \sigma) \bar{k}-\frac{1}{2}(1-4 \sigma-3 \zeta) \bar{c} \\
& -\frac{1}{2}(1-\zeta) \bar{e}
\end{aligned}
$$

$$
\begin{aligned}
\mu_{5}= & (1+2 \gamma+4 \hat{z}+6 \gamma \hat{z}+6 \sigma \hat{z}) \bar{b}-(\gamma+2 \hat{z}+3 \gamma \hat{z}) \bar{k} \\
& -\frac{1}{2}(1+2 \gamma+4 \hat{z}+6 \gamma \hat{z}-4 \sigma \hat{z}+\zeta) \bar{c} \\
& +\frac{1}{2}(\gamma+2 \hat{z}+3 \gamma \hat{z}+4 \sigma \hat{z}) \bar{e}
\end{aligned}
$$

$$
\begin{aligned}
\mu_{6}= & (1+2 \gamma+4 \hat{z}+6 \gamma \hat{z}+6 \sigma \hat{z}) \bar{a}+(\gamma+2 \hat{z}+3 \gamma \hat{z}+2 \sigma \hat{z}) \bar{k} \\
& -\frac{1}{2}(1+2 \gamma+4 \hat{z}+6 \gamma \hat{z}-4 \sigma \hat{z}+\zeta) \bar{d} \\
& -\frac{1}{2}(\gamma+2 \hat{z}+3 \gamma \hat{z}) \bar{e}
\end{aligned}
$$

$\nu_{1}=\hat{k}$,

$\nu_{2}=\hat{e}$

$$
\nu_{3}=\frac{1}{2}(2-2 \eta-\chi) \hat{d}-\frac{1}{2}(\eta+3 \chi) \hat{c}-\frac{1}{4}(\eta+2 \chi) \hat{e}-\frac{1}{2} \eta \hat{k},
$$

$$
\nu_{4}=\frac{1}{2}(2-2 \eta-\chi) \hat{b}-\frac{1}{2}(\eta+3 \chi) \hat{a}-\frac{1}{4} \eta \hat{e}-\frac{1}{2} \chi \hat{k},
$$

$$
\begin{aligned}
\nu_{5}= & \frac{1}{2}(2+\eta+3 \chi+6 \bar{z}+2 \eta \bar{z}+6 \chi \bar{z}) \hat{c} \\
& +\frac{1}{2}(2 \eta+\chi+2 \bar{z}+4 \eta \bar{z}+2 \chi \bar{z}) \hat{d}+\frac{1}{2}(\eta+2 \eta \bar{z}) \hat{k} \\
& +\frac{1}{4}(\eta+2 \chi+4 \bar{z}+2 \eta \bar{z}+4 \chi \bar{z}) \hat{e} \\
\nu_{6}= & \frac{1}{2}(2+\eta+3 \chi+6 \bar{z}+2 \eta \bar{z}+6 \chi \bar{z}) \hat{a}+\frac{1}{2}(2 \eta+\chi+2 \bar{z} \\
& +4 \eta \bar{z}+2 \chi \bar{z}) \hat{b}+\frac{1}{4}(\eta+2 \eta \bar{z}) \hat{e}+\frac{1}{2}(\chi+2 \bar{z}+2 \chi \bar{z}) \hat{k}
\end{aligned}
$$

Again, the full evolution equations are available from the authors upon request.

Furthermore, we note that if $\mu_{i}=\kappa \nu_{i}$ for all $i$ and constant $\kappa$, the system is symmetrizable hyperbolic using the energy norm argument of Ref. [13]. However, these conditions do not have to be met for the system to be well-posed. It is possible to construct a symmetrizer for any of the strongly hyperbolic systems. 


\section{G. Evolving with contravariant indices}

So far, we have written all of our fundamental variables with covariant indices. Alternatively, we could have defined the new variable

$$
D_{k}^{i j} \equiv \partial_{k} g^{i j}
$$

Note that $d_{k i j}=-D_{k i j}$. If we evolve $\left\{g^{i j}, P^{i j}, M_{k}{ }^{i j}\right\}$ instead of $\left\{g_{i j}, P_{i j}, M_{k i j}\right\}$, it would result in only trivial changes to the principal parts of the equations. The characteristic speeds would be unchanged, as would the nature of the hyperbolicity of the system, since the principal part of the metric evolution equation is zero (see Appendix B). The only changes would occur in the nonlinear terms of the evolution equations.

\section{H. Frittelli-Reula system}

We recover the system of Ref. [13] if we make the following choices for our parameters:

$$
\begin{aligned}
\{\sigma, \gamma, \zeta, \eta, \chi\} & =\left\{-\bar{\epsilon} \frac{1+3 \bar{\alpha}}{2}, \frac{2 \bar{\gamma}}{1+3 \bar{\beta}}, 1,4, \frac{-4 \bar{\alpha}}{1+3 \bar{\alpha}}\right\}, \\
\{\hat{z}, \hat{k}, \hat{a}, \hat{b}, \hat{c}, \hat{d}, \hat{e}\} & =\{\bar{\beta}, 1, \bar{\alpha}, 0,0,0,0\},
\end{aligned}
$$

where $\{\bar{\alpha}, \bar{\beta}, \bar{\gamma}, \bar{\epsilon}\}$ corresponds to $\{\alpha, \beta, \gamma, \epsilon\}$ in Ref. [13]. However, as pointed out in Ref. [31], this system is not symmetric hyperbolic unless the term $-2 h^{l(i} M^{j) k}{ }_{l, k}$ in Eq. (16) of Ref. [13] is replaced with $-2 h^{l(i} M_{k, l}^{j) k}$ by adding a term proportional to constraint (2.17). In our system this corresponds to changing $\zeta=1$ to $\zeta=-1$ in Eq. (2.55).

In Refs. [8,31,32], this correction has been made for the parameter choice $\{\bar{\alpha}, \bar{\beta}, \bar{\gamma}, \bar{\epsilon}\}=\left\{-1,-1,1, \frac{1}{2}\right\}$; we recover this system if we choose our parameters to be

$$
\begin{aligned}
\{\sigma, \gamma, \zeta, \eta, \chi\} & =\left\{\frac{1}{2},-1,-1,4,-2\right\}, \\
\{\hat{z}, \hat{k}, \hat{a}, \hat{b}, \hat{c}, \hat{d}, \hat{e}\} & =\{-1,1,-1,0,0,0,0\} .
\end{aligned}
$$

The system of Refs. [8,31,32] was further generalized in Ref. [27], where the constraints were used to modify the evolution equations in a manner similar to that in Sec. II D. We recover the system of Ref. [27] by choosing

$$
\begin{aligned}
\{\sigma, \gamma, \zeta, \eta, \chi\} & =\left\{\frac{1}{2},-\tilde{\gamma}, 2 \widetilde{\Theta}-1,4 \tilde{\eta},-2 \tilde{\eta}\right\}, \\
\{\hat{z}, \hat{k}, \hat{a}, \hat{b}, \hat{c}, \hat{d}, \hat{e}\} & =\{-1,1,-1,0,0,0,0\},
\end{aligned}
$$

where $\{\tilde{\gamma}, \widetilde{\Theta}, \tilde{\eta}\}$ correspond to $\{\gamma, \Theta, \eta\}$ in Ref. [27].

\section{Einstein-Christoffel system}

We recover the system of Ref. [22] if we make the following choices for our parameters:

$$
\begin{aligned}
\{\sigma, \gamma, \zeta, \eta, \chi\} & =\left\{\frac{1}{2}, 0,-1,4,0\right\}, \\
\{\hat{z}, \hat{k}, \hat{a}, \hat{b}, \hat{c}, \hat{d}, \hat{e}\} & =\{0,1,0,0,2,-2,0\} .
\end{aligned}
$$

This system is symmetrizable hyperbolic and has very simple principal parts

$$
\begin{aligned}
\hat{\partial}_{0} P_{i j} & \simeq-N g^{a b} \partial_{a} M_{b i j}, \\
\hat{\partial}_{0} M_{k i j} & \simeq-N \partial_{k} P_{i j} .
\end{aligned}
$$

Essentially this system is a set of six (one for each $\{i, j\}$ pair) coupled quasilinear scalar wave equations with nonlinear source terms.

\section{J. Generalized Einstein-Christoffel system: System (3)}

If we examine the principal part of system (2), and demand that $\mu_{1}=\nu_{1}=1$ and all other $\mu_{i}$ and $\nu_{i}$ vanish, we obtain a two-parameter system $\{\eta, \hat{z}\}$ that has the same simple wave-like form (2.59) as the Einstein-Christoffel system. This system is obtained by setting

$$
\begin{aligned}
\{\sigma, \gamma, \zeta, \eta, \chi\}= & \left\{\frac{1}{2}, \frac{-4+\eta}{2 \eta},-1, \eta, \frac{-4+\eta}{4}\right\}, \\
\{\hat{z}, \hat{k}, \hat{a}, \hat{b}, \hat{c}, \hat{d}, \hat{e}\}= & \left\{\hat{z}, 1, \frac{-4+\eta-12 \hat{z}+9 \eta \hat{z}}{2 \eta},\right. \\
& \left.\frac{4-\eta+12 \hat{z}-7 \eta \hat{z}}{2 \eta}, 2,-2,0\right\},
\end{aligned}
$$

where $\hat{z} \neq-\frac{1}{3}$ and $\eta \neq 0$. This system has physical characteristic speeds and is symmetrizable hyperbolic. The free parameter $\eta$ will affect the principal part of the constraint evolution equations, while the parameter $\hat{z}$ will affect only the nonlinear terms in the evolution equations and the constraint evolution equations. It is this system that we will explore numerically in Sec. III. The complete equations for this system are available upon request from the authors.

The characteristic eigenfields of this system are particularly simple, and can be obtained from Eq. (2.59) without the use of the lengthy decomposition procedure described in Appendix A. In a direction $\xi_{i}$, the eigenfields are

$$
\begin{aligned}
U_{i j}^{0} & \equiv g_{i j}, \\
U_{k i j}^{0} & \equiv M_{k i j}-\xi_{k} \xi^{l} M_{l i j}, \\
U_{i j}^{ \pm} & \equiv P_{i j} \pm \xi^{k} M_{k i j} .
\end{aligned}
$$

The $U^{0}$ quantities propagate along the normal to the time slice (coordinate speed $-\beta^{i}$ ), and the $U^{ \pm}$quantities propagate along the light cone (coordinate speed $-\beta^{i} \pm N \xi^{i}$ ). 


\section{NUMERICAL RESULTS}

In this section we present results from a numerical code that solves the evolution equations of system (3) in three spatial dimensions plus time. This code, which will be described in detail elsewhere [33], is a three-dimensional generalization of a spherically symmetric code discussed previously [34], and is based on pseudospectral collocation methods. Our code works in full three dimensions; we do not exploit any symmetries of the black hole solutions that we evolve.

In this paper, we will concern ourselves only with single black hole spacetimes. In this case, we solve the evolution equations in a spherical shell extending from inside the horizon to some artificial outer boundary. Although we use standard spherical polar coordinates $(r, \theta, \phi)$, we evolve the Cartesian components of our variables; this allows us to use scalar spherical harmonics $Y_{l m}(\theta, \phi)$ as angular basis functions for all quantities. We use Chebyshev polynomials as the basis functions in radius.

As described in Ref. [34], we use the method of lines in order to integrate forward in time with a fourth-order RungeKutta method. Boundary conditions are imposed by constructing the characteristic fields that propagate normal to the boundary, and imposing conditions only on those fields that propagate into the computational domain. Since all characteristic fields at the inner boundary are outgoing (into the hole), no boundary condition is needed there and none is imposed. At the outer boundary, we impose $\partial_{t} U^{-}=0$ on each of the characteristic fields $U^{-}$that is ingoing there. We use analytic initial data corresponding to time-independent slicings of a single black hole, and fix the gauge quantities $Q$ and $\beta^{i}$ to their analytic values for all time. Note that the constraint equations are not solved explicitly, but are instead used as a check on the accuracy of our numerical integrations.

\section{A. Einstein-Christoffel system}

Figure 1 shows the $l_{2}$ norm of a component of the momentum constraint for several evolutions of a Schwarzschild black hole using the Einstein-Christoffel system, which is equivalent to system (3) with $\eta=4$ and $\hat{z}=0$. Initially the fields are given analytically on a Painlevé-Gullstrand time slice [35-38]. Explicit formulas for our variables on the initial slice can be found in Ref. [34].

As is evident from Fig. 1, the constraint increases with time until the simulation terminates. The evolutions with higher radial resolution run longer, but increase at approximately the same rate. In addition, for a fixed resolution, we see no significant dependence on $\Delta t$, and for a fixed radial resolution and time step we see no significant dependence on the angular resolution. This suggests that the growth of the constraints may be due to an unphysical solution of the equations rather than a numerical instability. Numerical instabilities typically become worse when one increases the resolution or decreases the time step. In contrast, our results appear consistent with an unphysical solution of the equations that initially has a nonzero amplitude because of small numerical errors.

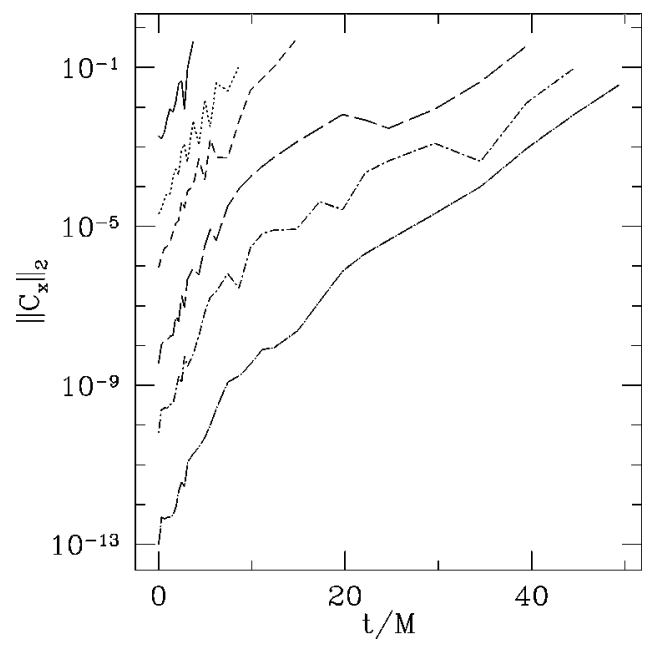

FIG. 1. Momentum constraint $\mathcal{C}_{x}$ vs time for evolutions of a Painlevé-Gullstrand time slicing of a Schwarzschild black hole using the Einstein-Christoffel system. Results are plotted for several radial resolutions ranging from $N_{r}=10$ to 40 , a fixed angular resolution $l=7$, and a fixed time resolution $\Delta t=0.015 M$. Higher radial resolutions correspond to smaller errors.

\section{B. Generalized Einstein-Christoffel system}

Because we suspected that the instability shown in Fig. 1 is related to the equations rather than the numerical method, we repeated the above evolutions for various values of the free parameters $\eta$ and $\hat{z}$, searching the two-dimensional parameter space for systems of evolution equations that might be better behaved. We found that for $\eta \simeq 4 / 33$ and $\hat{z} \simeq-1 / 4$, our numerical simulations ran for an order of magnitude longer than for the basic Einstein-Christoffel system. Typical results are plotted in Fig. 2. Although a growing mode is still present, its growth rate is much smaller than in Fig. 1, and the momentum constraint is less than $10^{-3}$ until approximately $600 \mathrm{M}$.

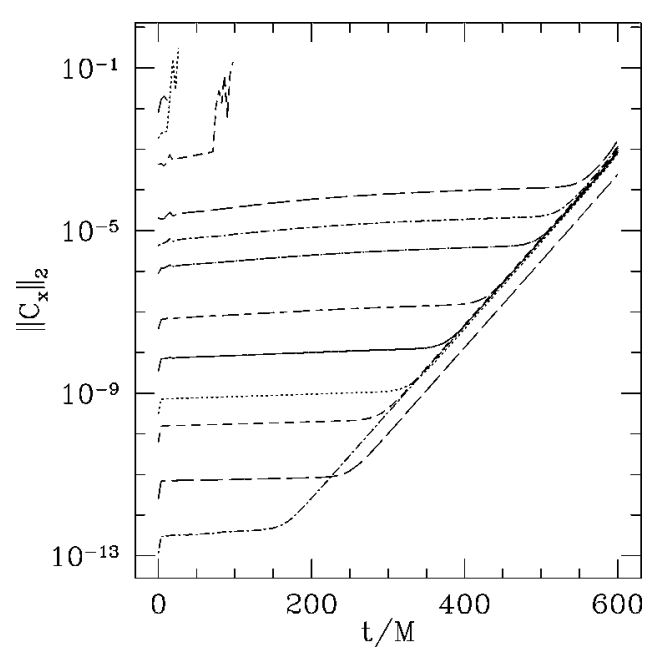

FIG. 2. Momentum constraint $\mathcal{C}_{x}$ vs time for the same evolutions shown in Fig. 1 except $\eta=4 / 33$ and $\hat{z}=-1 / 4$, and we plot more radial resolutions. If the outer boundary is moved out to $r=40 M$, the run time extends to $\sim 1300 M$ for the same accuracy. 
We see no evidence that the growth is due to a numerical instability. In contrast, the evolutions in Fig. 2 appear to converge to a well-defined solution. This solution is the sum of two components: a roughly time-independent component and an exponentially growing component. By extrapolating backwards along the growing component in Fig. 2, one can see that this component has magnitude $\sim 10^{-16}$ at $t=0$, which is on the order of machine roundoff error.

As in the Einstein-Christoffel case, we see no dependence on angular resolution or on $\Delta t$. Our results do depend upon the location of the outer boundary. In the evolution shown in Fig. 2, the spherical domain extends from $r=1.9 \mathrm{M}$ to $r$ $=11.9 \mathrm{M}$. Moving the outer boundary further out results in longer evolutions, increasing the run time from around $600 \mathrm{M}$ up to $1300 M$ with the outer boundary at $r=40 M$. Moving the outer boundary beyond $r=40 M$, however, does not have any effect.

In addition to Painlevé-Gullstrand slicings, we have run Kerr-Schild $[39,40]$ and harmonic-time [41,42] slicings of a Schwarzschild black hole with similar qualitative results. For example, using the parameters of Fig. 2 with a Kerr-Schild slicing as initial data, we were able to evolve up to $t$ $=500 \mathrm{M}$ with the outer boundary at $r=11.9 \mathrm{M}$, and up to $t$ $=900 \mathrm{M}$ with the outer boundary at $r=40 \mathrm{M}$. We have also evolved a Kerr black hole with $a=M / 2$ to $t=400 M$, with a spherical shell extending from $r=1.5 \mathrm{M}$ to $r=11.5 \mathrm{M}$.

\section{DISCUSSION}

We have constructed a 12-parameter family of hyperbolic formulations of Einstein's equations that is strongly hyperbolic for a wide range of the parameter space, and that includes the systems of Refs. [13] and [22]. By restricting ourselves to a two-parameter subset of these equations, we have demonstrated how the choice of parameters can have a dramatic effect upon the amount of time a numerical simulation of a black hole can run before being swamped by an unphysical solution.

Our runs with our best parameter choices appear to be limited only by the growth of constraint-violating modes which grow from the level of numerical roundoff errors. This is consistent with the results of Ref. [3], in which an unstable mode limited the evolution of a single Schwarzschild black hole to about $t=500 M$. A detailed comparison with their results is difficult to make, however, as they used a different set of evolution equations and a different numerical implementation, as well as different gauge conditions and boundary conditions. A key feature of their approach is to use a simple but stable boundary condition at the excision boundary, where physically no boundary condition should be needed. In our approach, we do not need to apply any boundary conditions at the excision boundary.

In our analysis of the hyperbolicity of the evolution equations we have assumed that the gauge variables (the shift vector and the densitized lapse) are given arbitrary functions that are independent of the dynamical variables (the metric, generalized extrinsic curvature, and generalized metric derivatives). In our numerical simulations, we achieved this by setting the gauge variables to their analytic values for the

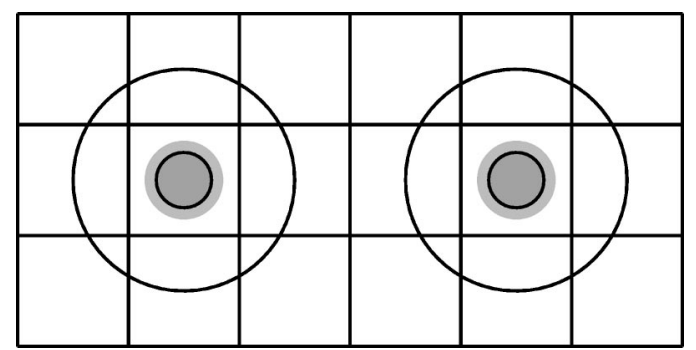

FIG. 3. Two-dimensional illustration of multiple computational domains that might be used to solve the binary black hole problem. Each black hole (shaded gray) is surrounded by a single domain in the shape of a spherical shell, with the inner boundary of the shell just inside the horizon. Multiple cubical domains overlap the spherical shells. There are 16 shown here in two dimensions; the two cubes that contain the black holes are excised.

particular time-independent slicing that we used as initial data. In order to perform a time-dependent evolution, however, it will be necessary to allow the gauge to change as the evolution proceeds. If this is done by choosing the gauge variables as functions of the dynamical variables, the hyperbolicity of the evolution equations may be affected. We are currently investigating how the imposition of more complicated gauge conditions can be done in a manner that does not spoil the hyperbolicity of the system. In Ref. [34], we were able to impose dynamical gauge conditions by solving elliptic equations for the shift and densitized lapse prior to each time step, and holding these quantities fixed during each step.

At present, we have no explanation as to why the particular choice of parameters used to produce Fig. 2 is so much better than the Einstein-Christoffel system. This choice was found empirically by running our code for various values of the parameters. It would be extremely useful to have some theoretical understanding of why one particular parameter choice behaves much better than another, as the cost of performing a parameter search on the full twelve-parameter system would be prohibitive.

Having found a system of equations and a numerical method capable of evolving a single black hole for a physically interesting length of time, we now plan to turn our attention to the evolution of a binary black hole system. For evolutions of two black holes with excised horizons it will be necessary to use multiple computational domains (see Fig. $3)$. Each domain is evolved independently except at the domain boundaries; there the incoming characteristic fields in each domain are filled with the corresponding outgoing characteristic fields from neighboring domains. We expect our computational method to be capable of evolving the binary system to times on the order of several hundred $M$ once the difficult problem of determining appropriate gauge conditions is solved. When we realize this, we will be able to simulate the last orbit or two prior to the plunge as well as the coalescence itself.

\section{ACKNOWLEDGMENTS}

We thank Harald Pfeiffer, Manuel Tiglio, and James W. York, Jr. for helpful discussions. This work was supported in 
part by NSF Grant Nos. PHY-9900672 and PHY-0084729 and NASA Grant No. NAG5-7264. Computations were performed on the National Computational Science Alliance SGI Origin 2000, and on the Wake Forest University Department of Physics IBM SP2 with support from an IBM SUR grant.

\section{APPENDIX A: HYPERBOLICITY}

To determine the characteristic speeds and eigenvectors of a system of the form of Eq. (2.25), we proceed in two steps. Instead of directly finding the eigenvalues and eigenvectors of $C \equiv A^{i} \xi_{i}$, we first construct a transformation $D$ such that $C^{\prime} \equiv D A^{i} \xi_{i} D^{-1}$ is independent of the direction $\xi_{i}$ and of the metric quantities $g_{i j}$. We then solve $C^{\prime} w_{i}=\lambda_{i} w_{i}$. The eigenvalues of the original matrix $C$ are $\lambda_{i}$, and the eigenvectors are $D^{-1} w_{i}$.

Transformation $D$ is the decomposition of each of the fundamental tensor (or tensor-like) quantities into its irreducible parts, as we now describe. Suppose $v \equiv D u$. Then if $u$ and $v$ are scalars, $D$ is the identity operator, $v=u$. For a vector quantity $u=V_{i}, D$ is defined by

$$
V_{i}=D^{-1} v=V_{i}^{(T)}+\xi_{i} V^{(L)}
$$

where the longitudinal and transverse parts of $V_{i}$ are given by

$$
V^{(L)} \equiv \xi^{m} V_{m}
$$

$$
V_{i}^{(T)} \equiv \perp_{i}{ }^{m} V_{m}
$$

where $\perp_{i j}$ is the projection operator:

$$
\perp_{i j} \equiv g_{i j}-\xi_{i} \xi_{j} .
$$

For a symmetric second-rank tensor $u=P_{i j}$,

$$
\begin{aligned}
P_{i j}= & D^{-1} v \\
= & P_{i j}^{(T T S)}+2 \xi_{(i} P_{j)}^{(L T)}+\frac{1}{2}\left(3 \xi_{i} \xi_{j}-g_{i j}\right) P^{(L L)} \\
& +\frac{1}{2}\left(g_{i j}-\xi_{i} \xi_{j}\right) P,
\end{aligned}
$$

where

$$
\begin{aligned}
P & \equiv g^{m n} P_{m n}, \\
P^{(L L)} & \equiv \xi^{m} \xi^{n} P_{m n}, \\
P_{i}^{(L T)} & \equiv \xi^{m} \perp_{i}^{n} P_{m n}, \\
P_{i j}^{(T T S)} & \equiv\left(\perp_{(i}^{m} \perp_{j)}^{n}-\frac{1}{2} \perp_{i j} \perp^{m n}\right) P_{m n} .
\end{aligned}
$$

For a third-rank object $u=M_{k i j}$, symmetric on its last two indices,

$$
\begin{aligned}
M_{k i j}= & D^{-1} v \\
= & M_{k i j}^{(T T T)}+2 \xi_{(i} M_{j) k}^{(T T L s)}+2 \xi_{(i} M_{j) k}^{(T T L a)}+\xi_{k} M_{i j}^{(L T T)}+\frac{1}{4} M_{k}^{(T L L)}\left(7 \xi_{i} \xi_{j}-3 g_{i j}\right)+\frac{1}{2} M_{(i}^{(T L L)}\left(g_{j) k}-\xi_{j)} \xi_{k}\right) \\
& +\frac{1}{2} M_{k}^{(L L T)}\left(g_{i j}-\xi_{i} \xi_{j}\right)+M_{(i}^{(L L T)}\left(3 \xi_{j} \xi_{k}-g_{j) k}\right)+\frac{3}{4} M_{k}^{(T R R)}\left(g_{i j}-\xi_{i} \xi_{j}\right)+\frac{1}{2} M_{(i}^{(T R R)}\left(\xi_{j)} \xi_{k}-g_{j) k}\right) \\
& +\frac{1}{2} M_{k}^{(R R T)}\left(\xi_{i} \xi_{j}-g_{i j}\right)+M_{(i}^{(R R T)}\left(g_{j) k}-\xi_{j)} \xi_{k}\right)+\frac{1}{2} M^{(L L L)}\left(5 \xi_{k} \xi_{i} \xi_{j}-\xi_{k} g_{i j}-2 g_{k(i} \xi_{j)}\right)+\frac{1}{2} M^{(L R R)}\left(\xi_{k} g_{i j}-\xi_{k} \xi_{i} \xi_{j}\right) \\
& +M^{(R R L)}\left(g_{k(i} \xi_{j)}-\xi_{k} \xi_{i} \xi_{j}\right)
\end{aligned}
$$

where

$$
\begin{aligned}
& M^{(R R L)} \equiv g^{c a} \xi^{b} M_{c a b}, \\
& M^{(L R R)} \equiv g^{a b} \xi^{c} M_{c a b}, \\
& M^{(L L L)} \equiv \xi^{c} \xi^{a} \xi^{b} M_{c a b}, \\
& M_{i}^{(R R T)} \equiv g^{c a} \perp_{i}{ }^{b} M_{c a b}, \\
& M_{i}^{(T R R)} \equiv g^{a b} \perp_{i}{ }^{c} M_{c a b}, \\
& M_{i}^{(L L T)} \equiv \xi^{c} \xi^{a} \perp_{i}{ }^{b} M_{c a b}, \\
& M_{i}^{(T L L)} \equiv \xi^{a} \xi^{b} \perp_{i}{ }^{c} M_{c a b},
\end{aligned}
$$




$$
\begin{aligned}
& M_{i j}^{(L T T)} \equiv \xi^{c}\left(\perp_{i}^{a} \perp_{j}^{b}-\frac{1}{2} \perp_{i j} \perp^{a b}\right) M_{c a b}, \\
& M_{i j}^{(T T L s)} \equiv \xi^{b}\left(\perp_{(i}{ }^{c} \perp_{j)}{ }^{a}-\frac{1}{2} \perp_{i j} \perp^{c a}\right) M_{c a b}, \\
& M_{i j}^{(T T L a)} \equiv \xi^{b} \perp_{[i}{ }^{c} \perp_{j]}{ }^{a} M_{c a b}, \\
& M_{k i j}^{(T T T)} \equiv\left[\perp_{k}{ }^{c} \perp_{i}{ }^{a} \perp_{j}{ }^{b}-\frac{1}{4} \perp_{i j}\left(3 \perp^{a b} \perp_{k}{ }^{c}-\perp^{c a} \perp_{k}{ }^{b}-\perp^{c b} \perp_{k}{ }^{a}\right)\right. \\
& -\frac{1}{4} \perp_{k j}\left(3 \perp^{c b} \perp_{i}{ }^{a}-\perp^{c a} \perp_{i}{ }^{b}-\perp^{a b} \perp_{i}{ }^{c}\right) \\
& \left.-\frac{1}{4} \perp_{k i}\left(3 \perp^{c a} \perp_{j}{ }^{b}-\perp^{a b} \perp_{j}{ }^{c}-\perp^{c b} \perp_{j}{ }^{a}\right)\right] M_{c a b} .
\end{aligned}
$$

Finally for a four-index object $\mathcal{C}_{k l i j}$, symmetric on its last two indices and antisymmetric on its first two indices,

$$
\begin{aligned}
\mathcal{C}_{k l i j}= & D^{-1} v \\
= & \mathcal{C}_{k l i j}^{(T F)}+\frac{3}{5} \mathcal{C}_{k l}^{(T T R R a)} g_{i j}+\frac{4}{5} \mathcal{C}_{k l}^{(R T T R a)} g_{i j}+\frac{2}{5}\left(\mathcal{C}_{i[k}^{(T T R R a)} g_{l] j}+\mathcal{C}_{j[k}^{(\text {TTRRa })} g_{l] i}\right)+\frac{6}{5}\left(\mathcal{C}_{i[k}^{(R T T R a)} g_{l] j}+\mathcal{C}_{j[k}^{(R T T R a)} g_{l] i}\right) \\
& -\frac{2}{3}\left(\mathcal{C}_{i[k}^{(R T T R s)} g_{l] j}+\mathcal{C}_{j[k}^{(R T T R s)} g_{l] i}\right)-\frac{6}{5} \mathcal{C}_{[k}^{(L T R R)} \xi_{l]} g_{i j}+\frac{4}{5} \mathcal{C}_{[k}^{(L T R R)} g_{l](j)} \xi_{i)}+\frac{4}{5} \mathcal{C}_{(i}^{(L T R R)} g_{j)[k} \xi_{l]}-\frac{4}{5} \mathcal{C}_{[k}^{(R L T R)} \xi_{l]} g_{i j} \\
= & \mathcal{C}_{k l i j}^{(T F)}+\frac{3}{5} \mathcal{C}_{k l}^{(T T R R a)} g_{i j}+\frac{4}{5} \mathcal{C}_{k l}^{(R T T R a)} g_{i j}+\frac{2}{5}\left(\mathcal{C}_{i[k}^{(T T R R a)} g_{l] j}+\mathcal{C}_{j[k}^{(T T R R a)} g_{l] i}\right)+\frac{6}{5}\left(\mathcal{C}_{i[k}^{(R T T R a)} g_{l] j}+\mathcal{C}_{j[k}^{(R T T R a)} g_{l] i}\right) \\
& -\frac{2}{3}\left(\mathcal{C}_{i[k}^{(R T T R s)} g_{l] j}+\mathcal{C}_{j[k}^{(R T T R s)} g_{l] i}\right)-\frac{6}{5} \mathcal{C}_{[k}^{(L T R R)} \xi_{l]} g_{i j}+\frac{4}{5} \mathcal{C}_{[k}^{(L T R R)} g_{l](j)} \xi_{i j}+\frac{4}{5} \mathcal{C}_{(i}^{(L T R R)} g_{j)[k} \xi_{l]}-\frac{4}{5} \mathcal{C}_{[k}^{(R L T R)} \xi_{l]} g_{i j} \\
& +\frac{8}{15} \mathcal{C}_{[k}^{(R L T R)} g_{l](j)} \xi_{i)}+\frac{28}{15} \mathcal{C}_{(i}^{(R L T R)} g_{j)[k} \xi_{l]}+\frac{4}{5} \mathcal{C}_{[k}^{(R T L R)} \xi_{l]} g_{i j}-\frac{28}{15} \mathcal{C}_{[k}^{(R T L R)} g_{l](j)} \xi_{i)}-\frac{8}{15} \mathcal{C}_{(i}^{(R T L R)} g_{j)[k} \xi_{l]}+2 \xi_{(i} g_{j)[k} \xi_{l]} \mathcal{C}^{(R L L R)},
\end{aligned}
$$

where

$$
\begin{aligned}
& \mathcal{C}^{(R L L R)} \equiv g^{c b} \xi^{d} \xi^{a} \mathcal{C}_{c d a b}, \\
& \mathcal{C}_{i}^{(R T L R)} \equiv g^{c b} \xi^{a} \perp_{i}{ }^{d} \mathcal{C}_{c d a b}, \\
& \mathcal{C}_{i}^{(R L T R)} \equiv g^{c b} \xi^{d} \perp_{i}{ }^{a} \mathcal{C}_{c d a b}, \\
& \mathcal{C}_{i}^{(\operatorname{LTRR})} \equiv g^{a b} \xi^{c} \perp_{i}{ }^{d} \mathcal{C}_{c d a b}, \\
& \mathcal{C}_{i j}^{(R T T R s)} \equiv g^{c b}\left(\perp_{(i}{ }^{d} \perp_{j)}{ }^{a}-\frac{1}{2} \perp_{i j} \perp^{d a}\right) \mathcal{C}_{c d a b}, \\
& \mathcal{C}_{i j}^{(R T T R a)} \equiv g^{c b} \perp_{[i}{ }^{d} \perp_{j]}{ }^{a} \mathcal{C}_{c d a b}, \\
& \mathcal{C}_{i j}^{(T T R R a)} \equiv g^{a b} \perp_{[i}{ }^{c} \perp_{j]}{ }^{d} \mathcal{C}_{c d a b}, \\
& \mathcal{C}_{k l i j}^{(T F)} \equiv\left(g_{k}{ }^{c} g_{l}{ }^{d} g_{i}{ }^{a} g_{j}{ }^{b}-\frac{28}{15} g^{c b} g^{a}{ }_{(i} g_{j)[k} g_{l]}{ }^{d}+\frac{8}{15} g^{c b} g^{d}{ }_{(i} g_{j)[k} g_{l]}{ }^{a}\right. \\
& \left.-\frac{4}{5} g^{a b} g^{d}{ }_{(i} g_{j)[k} g_{l]}{ }^{c}-\frac{4}{5} g_{i j} g^{c b} g_{[k}^{d} g_{l]}{ }^{a}-\frac{3}{5} g_{i j} g^{a b} g_{k}{ }^{c} g_{l}{ }^{d}\right) \mathcal{C}_{c d a b} .
\end{aligned}
$$


Strictly speaking, Eqs. (A6) and (A8) are not complete irreducible decompositions. However, they are sufficient for our purposes.

If $u$ consists of several tensor (or tensorlike) objects, then the effect of $D$ is to transform each object independently according to the above definitions. In matrix language, this means that $D$ is block diagonal.

\section{APPENDIX B: CHANGE OF VARIABLES AND HYPERBOLICITY}

In this section we show that for a system of the form of Eq. (2.25), a change of variables [such as the transformation from system (1) to system (2), or the raising and lowering of tensor indices of fundamental variables] does not change either the characteristic speeds or whether the system is strongly hyperbolic, provided that the following conditions are met:

(1) The change of variables is linear in all dynamical variables except possibly the metric.

(2) The change of variables is invertible.

(3) Time and space derivatives of the metric can be written as a sum of only non-principal terms [for example, using Eqs. (2.12) and (2.13)].

For a system of the form of Eq. (2.25), we choose an arbitrary direction $\xi_{i}$ and we define the matrix $C$ according to Eq. (2.26). The system has $k$ characteristic speeds $\lambda^{(k)}$ and eigenvectors $w^{(k)}$ that obey

$$
C w^{(k)}=\lambda^{(k)} w^{(k)} .
$$

If $M$ is the matrix whose columns are the eigenvectors $w^{(k)}$, then strong hyperbolicity is equivalent to $\operatorname{det} M \neq 0$, with all $\lambda^{(k)}$ real.

Now consider a change of variables $v=T u$, where $T$ is a matrix. If we multiply Eq. (2.25) on the left by $T$, we obtain

$$
\begin{aligned}
\hat{\partial}_{0} v+T A^{i} T^{-1} \partial_{i} v & =T F+\left(\hat{\partial}_{0} T\right) u+T A^{i} T^{-1}\left(\partial_{i} T\right) u \\
& =F^{\prime} .
\end{aligned}
$$

In the last step, we have used property (1) above to rewrite $\partial_{i} T$ and $\hat{\partial}_{0} T$ in terms of derivatives of the metric, and we have used property (3) to eliminate these derivatives, absorbing the resulting non-principal terms into the new right-hand side $F^{\prime}$.

The characteristic matrix for Eq. (B2) in the direction $\xi_{i}$ is $C^{\prime} \equiv T A^{i} T^{-1} \xi_{i}$. Note that

$$
C^{\prime} T w^{(k)}=T A^{i} T^{-1} \xi_{i} T w^{(k)}=T A^{i} \xi_{i} w^{(k)}=\lambda^{(k)} T w^{(k)},
$$

so Eqs. (B2) and (2.25) have the same characteristic speeds $\lambda^{(k)}$, and the eigenvectors of Eq. (B2) are $T w^{(k)}$.

Furthermore, the matrix of eigenvectors for Eq. (B2) is $M^{\prime}=(T M)^{T}$, so

$$
\operatorname{det} M^{\prime}=\operatorname{det}(T M)^{T}=\operatorname{det} T \operatorname{det} M .
$$

If the transformation $T$ is invertible, $\operatorname{det} M^{\prime} \neq 0$ if and only if $\operatorname{det} M \neq 0$, so Eq. (B2) is strongly hyperbolic if and only if Eq. (2.25) is hyperbolic.
[1] S. Brandt et al., Phys. Rev. Lett. 85, 5496 (2000).

[2] M. Alcubierre et al., gr-qc/0012079.

[3] M. Alcubierre and B. Brügmann, Phys. Rev. D 63, 104006 (2001).

[4] M.A. Scheel et al., Phys. Rev. D 58, 044020 (1998).

[5] T.W. Baumgarte and S.L. Shapiro, Phys. Rev. D 59, 024007 (1999).

[6] M. Alcubierre et al., Phys. Rev. D 62, 124011 (2000).

[7] M. Alcubierre et al., Phys. Rev. D 62, 044034 (2000).

[8] S. Frittelli and O. Reula, Commun. Math. Phys. 166, 221 (1994).

[9] Y. Choquet-Bruhat and J.W. York, Jr., C. R. Acad. Sci., Ser. I: Math. A321, 1089 (1995).

[10] A. Abrahams, A. Anderson, Y. Choquet-Bruhat, and J.W. York, Jr., Phys. Rev. Lett. 75, 3377 (1995).

[11] C. Bona, J. Massó, E. Seidel, and J. Stela, Phys. Rev. Lett. 75, 600 (1995).

[12] M.H.P.M. van Putten and D.M. Eardley, Phys. Rev. D 53, 3056 (1996).

[13] S. Frittelli and O.A. Reula, Phys. Rev. Lett. 76, 4667 (1996).

[14] H. Friedrich, Class. Quantum Grav. 13, 1451 (1996).

[15] F.B. Estabrook, R.S. Robinson, and H.D. Wahlquist, Class. Quantum Grav. 14, 1237 (1997)

[16] M.S. Iriondo, E.O. Leguizamon, and O.A. Reula, Phys. Rev. Lett. 79, 4732 (1997).

[17] A. Anderson, Y. Choquet-Bruhat, and J.W. York, Jr., Top.
Meth. Nonlin. Anal. 10, 353 (1997).

[18] M.A.G. Bonilla, Class. Quantum Grav. 15, 2001 (1998).

[19] G. Yoneda and H. Shinkai, Phys. Rev. Lett. 82, 263 (1999).

[20] M. Alcubierre, B. Brügmann, M. Miller, and W.-M. Suen, Phys. Rev. D 60, 064017 (1999).

[21] S. Frittelli and O.A. Reula, J. Math. Phys. 40, 5143 (1999).

[22] A. Anderson and J.W. York, Jr., Phys. Rev. Lett. 82, 4384 (1999).

[23] H. Friedrich and A. Rendall, in Einstein's Field Equations and their Physical Implications, Lecture Notes in Physics, edited by B.G. Schmidt (Springer-Verlag, Berlin, 2000), pp. 127223.

[24] G. Yoneda and H. Shinkai, Int. J. Mod. Phys. D 9, 13 (2000).

[25] H. Shinkai and G. Yoneda, Class. Quantum Grav. 17, 4799 (2000).

[26] G. Yoneda and H. Shinkai, Class. Quantum Grav. 18, 441 (2001).

[27] S.D. Hern, Ph.D. thesis, University of Cambridge, 1999, gr-qc/ 0004036.

[28] R. Arnowitt, S. Deser, and C. W. Misner, in Gravitation: An Introduction to Current Research, edited by L. Witten (Wiley, New York, 1962), pp. 227-265.

[29] J.W. York, Jr., in Sources of Gravitational Radiation, edited by L.L. Smarr (Cambridge University Press, Cambridge, England, 1979), pp. 83-126.

[30] H.-O. Kreiss and J. Lorenz, Initial-Boundary Value Problems 
and the Navier-Stokes Equations (Academic Press, San Diego, 1989).

[31] J.M. Stewart, Class. Quantum Grav. 15, 2865 (1998).

[32] O. Brodbeck, S. Frittelli, P. Hübner, and O.A. Reula, J. Math. Phys. 40, 909 (1999).

[33] L.E. Kidder, M.A. Scheel, H.P. Pfeiffer, and S.A. Teukolsky (unpublished).

[34] L.E. Kidder et al., Phys. Rev. D 62, 084032 (2000).

[35] C. Gundlach and P. Walker, Class. Quantum Grav. 16, 991
(1999).

[36] P. Painlevé, C. R. Acad. Sci. Paris, 173, 677 (1921).

[37] A. Gullstrand, Ark. Mat., Astron. Fys. 16, 1 (1922).

[38] K. Martel and E. Poisson, Am. J. Phys. 69, 476 (2001).

[39] R.L. Marsa and M.W. Choptuik, Phys. Rev. D 54, 4929 (1996).

[40] R.A. Matzner, M.F. Huq, and D. Shoemaker, Phys. Rev. D 59, 024015 (1999).

[41] C. Bona and J. Massó, Phys. Rev. D 38, 2419 (1988).

[42] G.B. Cook and M.A. Scheel, Phys. Rev. D 56, 4775 (1997). 